\title{
Possible effect of collective modes in zero magnetic field transport in an electron-hole bilayer
}

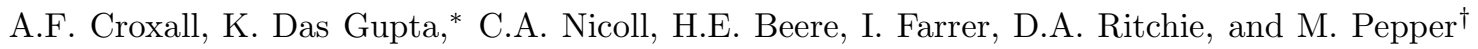 \\ Cavendish Laboratory, University of Cambridge, J.J. Thomson Avenue, Cambridge CB3 OHE, UK.
}

\begin{abstract}
We report single layer resistivities of 2-dimensional electron and hole gases in an electron-hole bilayer with a $10 \mathrm{~nm}$ barrier. In a regime where the interlayer interaction is stronger than the intralayer interaction, we find that an insulating state $(d \rho / d T<0)$ emerges at $T \sim 1.5 \mathrm{~K}$ or lower, when both the layers are simultaneously present. This happens deep in the "metallic" regime, even in layers with $k_{F} l>500$, thus making conventional mechanisms of localisation due to disorder improbable. We suggest that this insulating state may be due to a charge density wave phase, as has been expected in electron-hole bilayers from the Singwi-Tosi-Land-Sjölander approximation based calculations of L. Liu et al [Phys. Rev. B, 53, 7923 (1996)]. Our results are also in qualitative agreement with recent Path-Integral-Monte-Carlo simulations of a two component plasma in the low temperature regime [ P. Ludwig et al. Contrib. Plasma Physics 47, No. 4-5, 335 (2007)]
\end{abstract}

PACS numbers: 73.40.Kp, 73.43.Lp

Keywords: electron-hole, bilayer, coulomb drag, charge density wave

\section{INTRODUCTION}

The total energy of a system of electrons can be thought of as the sum total of the kinetic energies of the free particles and their potential energies due to mutual Coulomb interaction. The relative importance of the two contributions is measured by the parameter $r_{s}=E_{e e} / E_{f}$ (where $E_{e e}=e^{2} \sqrt{(\pi N)} / 4 \pi \kappa \epsilon_{0}$ and $E_{f}=\pi \hbar^{2} N / m_{e f f}$ in 2-dimensions, with $\mathrm{N}$ electrons per unit area). $r_{s}$ is a crucial parameter (apart from disorder) that governs the behaviour of the system to a large extent. For example it is known that a system of electrons or holes would be "gas like" at $r_{s} \approx 1$, "liquid like" at $r_{s} \approx 10$ and possibly a solid "Wigner crystal" at $r_{s} \approx 100$, provided disorder driven localisation does not dominate and pre-empt the observable effects of interaction. Confining a large number of particles in a small area makes the inter-particle spacing small and hence the Coulomb repulsion large, but the kinetic energy of the particles increases even faster - making $r_{s}$ smaller. This somewhat counter-intuitive fact is a straightforward consequence of Fermi statistics and is true in all dimensions.

Let us now consider two parallel layers of electrons or holes with $10^{11} \mathrm{~cm}^{-2}$ electrons in each. As they are brought closer to each other the particles in one layer not only interact with others in the same layer but also with those in the other layer. The inter-particle spacing in the same layer stays fixed and is about $30 \mathrm{~nm}$. It is possible (though highly non-trivial) to make the distance between the two layers about $10 \mathrm{~nm}$ without their wavefunctions beginning to overlap. $10 \mathrm{~nm}$ is approximately the excitonic Bohr radius in Gallium Arsenide (GaAs) and is an important length scale. We thus get an electron to "see" another electron (or hole) only $10 \mathrm{~nm}$ away, without paying the kinetic (Fermi) energy cost, because the two layers continue to be two separate Fermi systems. As a consequence bilayer systems can give rise to interaction-driven phases that are not possible in single layers. The case of attractive interaction (electron-hole) is more interesting. A rich phase diagram is anticipated for the ground state of a spatially separated electron-hole bilayer (EHBL) that includes a superfluid $1,2,3,4,5$, charge density waves (CDW), Wigner crystals (WC) $)^{6.7 .8}$, an excitonic supersolid ${ }^{\underline{9}}$ and a possible crossover from a Bose-condensate to BardeenCooper-Schreiffer type state $\stackrel{10}{ }$. Recent techniques for making independent ohmic contacts to 2-dimensional electron gases (2DEG) and 2-dimensional hole gases (2DHG) 10-20nm apart have enabled transport measurements down to millikelvin temperatures $11,12,13$. This has greatly extended the range of densities and temperatures that could be explored in the first attempt to measure transport in EHBLs 14 .

Quite naturally the initial experiments were focussed on measuring the Coulomb drag in these bilayers ${ }^{15.16}$. These results strongly suggest the emergence of a non Fermi-liquid phase at temperatures below $T \sim 1 K$. The scattering rate between the electrons and holes (that is measured in Coulomb drag experiments) is not determined by the bare Coulomb potential, as the inter-particle interaction is screened and it is the dielectric function $\epsilon(q, \omega)$, in the presence of interactions (and possible pairing fluctuations $\underline{4}^{4}$ ) that determines the measured Coulomb drag. On the other hand the dominant contribution to the single layer resistance comes from the scattering due to residual charged background impurities/traps and the remote ionised dopants (if any). The bare Coulomb potential due to these is screened by the same dielectric function. Thus it is worth asking the question: Does the interlayer interaction also bring about some distinct feature in the single layer resistances? This is the central question we investigate experimentally in this paper. A connection between drag and single layer resistance was earlier investigated in the context of in-plane magnetoresistance and magnetodrag in hole gases $\frac{17}{7}$. There is however a difference between the screening of interlayer electron- 
hole interactions and impurity potentials. The electrons and holes are mobile and the impurities are static. The potentials due to electrons/holes change with time and one needs to consider dynamic screening. On the other hand static screening $\epsilon(q, \omega=0)$ is sufficient to account for the effect of ionised impurities. Thus the Coulomb drag is sensitive to more features in the screening function (such as plasmon modes). Also, it is easy to imagine situations where the Coulomb drag and single layer resistivities would have opposite temperature dependences. For example, if the layers are individually insulating, then the resistivity of the layer will increase as the temperature is decreased, but the Coulomb drag will go to zero monotonically at low temperatures. While we are aware of these distinctions between drag and single layer resistance, we find striking features in the temperature dependence of the single layer resistivities of both layers. These features are unambiguously attributable to the presence of the other layer and hence electron-hole interaction only.

\section{DEVICES AND EXPERIMENTAL METHODS}

In our experiments the electron and hole densities $(n, p)$ were varied between $4 \times 10^{10} \mathrm{~cm}^{-2}$ and $1.6 \times 10^{11} \mathrm{~cm}^{-2}$, separated by a $10 \mathrm{~nm} \mathrm{Al}_{0.9} \mathrm{Ga}_{0.1} \mathrm{As}$ barrier. In this regime the inter-layer interaction is comparable to or even stronger than the intra-layer interaction. Several important length scales in the problem are very close to each other. Considering a typical density of $1 \times 10^{11} \mathrm{~cm}^{-2}$, the inverse Fermi wavevector is $k_{F}^{-1}=1 / \sqrt{2 \pi n} \approx 13 \mathrm{~nm}$. The hole quantum well is $20 \mathrm{~nm}$ wide. The peak-to-peak distance of the wavefunctions is $d \approx 25 \mathrm{~nm}$. For the lowest density $\left(n=p=4 \times 10^{10} \mathrm{~cm}^{-2}\right)$, we have $k_{F} d=1.2$. At the same time, the (conduction band) excitonic Bohr radius in GaAs is $a_{B} \approx 10 \mathrm{~nm}$. The interaction parameter for the electrons, $r_{s}$ (ratio of the interparticle Coulomb energy to the Fermi energy) at the lowest density is 2.8 and is $\approx 12$ for the holes. Thus, we reach a regime $d \sim r_{s} a_{B}$ where excitonic phases ${ }^{1,7}$ and density modulated phases $\frac{6,8}{6}$ have been predicted.The Molecular Beam Epitaxy growth, fabrication process and operation of these devices have been described in detail earlier ${ }^{11,13}$, so we do not describe them here. Two samples (Device D \& E) fabricated from the same wafer (ID:A4268) were used in this study and gave very similar results. (A third sample also gave similar results.) Coulomb drag data from device D was reported earlier ${ }^{15}$.

The complexity of these samples, at present, stands in the way of integrating coplaner waveguides and performing high frequency $(\sim 100 \mathrm{MHz})$ experiments to probe the "pinning modes" of density modulated phases. Such experiments have been done on bilayer hole gases, for
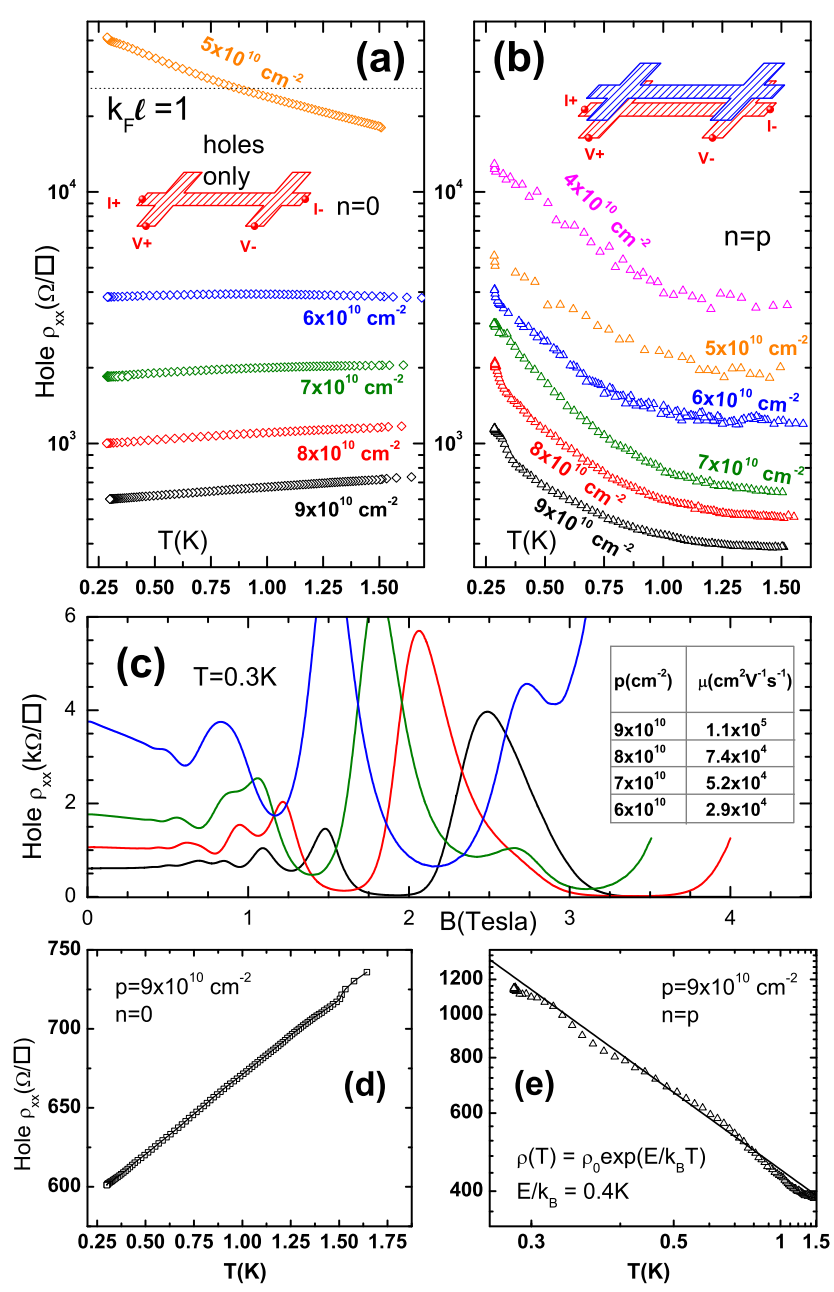

FIG. 1: (Color online)Data from device E, measured in a pumped $\mathrm{He}^{3}$ cryostat. (a) $\rho_{x x}(T)$ of the hole layer, when no electrons are present. (b) $\rho_{x x}(T)$ of the hole layer with electrons present. Exactly the same current and voltage contacts, measuring current $(\sim 10 \mathrm{nA})$ and frequency $(7 \mathrm{~Hz})$ were used for consistency. (c) Shubnikov de-Haas data corresponding to the traces in (a) showing normal 2DHG behaviour. The lowest trace corresponds to $p=9 \times 10^{10} \mathrm{~cm}^{2}$. (d) "Metallic" behaviour of the holes at $p=9 \times 10^{10} \mathrm{~cm}^{2}$, with $n=0$. (e)Near exponential rise of $\rho_{x x}(T)$, at $p=9 \times 10^{10} \mathrm{~cm}^{2}$ with $n=p$. Note that the figure is plotted in a log vs $\frac{1}{T}$ scale.

example $\frac{18}{}$, at the cost of sacrificing independent contacts and independent tuning of the layer densities. These experiments, if they can be done on EHBLs in future, would certainly provide valuable data. However we show that even dc transport in EHBLs (with its limitations), yields results strongly suggestive of the appearance of CDW or WC phases in EHBLs.

\section{RESULTS}

We first show the temperature dependence of the resistivity of the holes, when no electrons are present (Fig. 
[1]). This was achieved by keeping the interlayer bias voltage below the threshold ( $V_{\text {eh }} \approx 1.55 \mathrm{~V}$ ) for accumulation of electrons. The density of the holes was controlled by using the backgate. The data shows features typical of numerous studies on Si-MOSFETS and GaAs based single layer 2DEGs or 2DHGs in the context of the 2D Metal-Insulator transition (MIT) problem. At low densities $\left(p \leq 5 \times 10^{10} \mathrm{~cm}^{-2}\right)$ the traces are clearly insulating $(d \rho / d T<0)$, and turn metallic $(d \rho / d T>0)$ at higher densities. The crossover happens as expected near $\rho_{x x} \approx h / e^{2}$ or equivalently when $k_{F} l \approx 1$. The central result of this paper is the strikingly different behaviour of the 2DHG when it sees the 2DEG. Fig. 1o, where we plot the hole resistivity for several equal electron and hole densities, shows that even the traces which were metallic in Fig. 1a (e.g. $p=9 \times 10^{10} \mathrm{~cm}^{-2}$ in Fig. 1k) turn very clearly insulating by $T \sim 1 \mathrm{~K}$ even at $k_{F} l>50$. We will show later that $n=p$ is not essential for this insulating behaviour to appear. Simple arguments based on localisation due to background impurities/dopants/defects cannot account for this because the 2DHG (without the 2DEG) would see exactly the same charged background impurities. This is the key point that allows us to attribute this effect to electron-hole interactions. In fact if we look at the high temperature end of the traces $(T \approx 1.5 \mathrm{~K})$ we find that the resistivity of the 2DHG actually decreases significantly as the electrons are introduced. For the $p=6 \times 10^{10} \mathrm{~cm}^{-2}$ trace, $\rho_{x x}(n=0)=3808 \Omega / \square$, whereas $\rho_{x x}(n=p)=1203 \Omega / \square$ at $T=1.5 \mathrm{~K}$ (compare Fig. 1(a) and 1(b)). This is a very significant (threefold) increase in mobility and consistent with the findings of Morath et al in EHBLs with $30 \mathrm{~nm}$ barriers 19 . The effect is much stronger here, most likely because of the much thinner barrier used in this study. The increase in mobility at the high temperature end is most likely brought about by the extra screening of charged impurities due to the 2DEG and the "squeezing" of the hole wavefunction itself. The Coulomb drag was also measured for both the samples (data not shown) and at $T=1.5 \mathrm{~K}$ the values obtained are consistent with a $T^{2}$ behaviour. Below $T \approx 1 \mathrm{~K}$ both samples show a strong deviation from $T^{2}$ behaviour, as reported recently by us and Seamons et $a \mathbb{1 5}^{15,16}$. (The $T^{2}$ behaviour can be well understood within Fermi-liquid theories, but the strong non-monotonic deviations cannot be.) But a simple Mathiessen's rule based addition of two contributions due to Coulomb drag and impurity scattering cannot explain an increase in mobility. At the same time (for $n=p=9 \times 10^{10} \mathrm{~cm}^{-2}$ ) the measured Coulomb drag at $T<1 \mathrm{~K}$ is much smaller than the change in single layer resistivity (of the 2DHG) brought about by the presence of the 2DEG. Simple (incoherent) addition of various scattering rates no longer appears to be able to account for the observed magnitude of the effect.

If we now consider the behaviour of the electron layer, at $n=p=9 \times 10^{10} \mathrm{~cm}^{-2}$, the situation appears even more striking because the insulating behaviour can be easily seen at $k_{F} l>500$. Note that (in Fig. 2) although

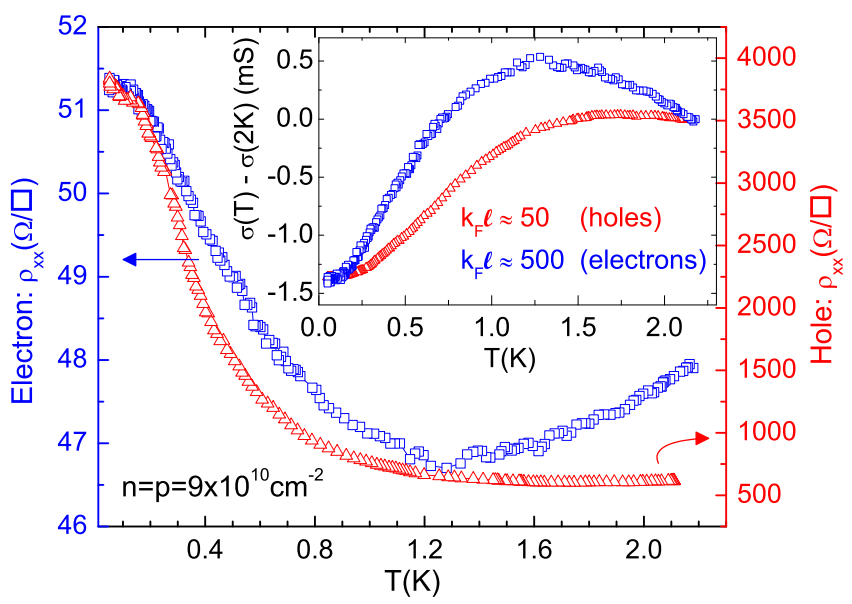

FIG. 2: (Color online) A comparison of the electron(blue) and hole (red) resistivity data from device $\mathrm{D}$, measured in a dilution refrigerator with base temperature of $\approx 50 \mathrm{mK}$. At this density were $\mu_{e}=1.5 \times 10^{6} \mathrm{~cm}^{2} \mathrm{~V}^{-1} \mathrm{~s}^{-1} \& \mu_{h}=1.1 \times$ $10^{5} \mathrm{~cm}^{2} \mathrm{~V}^{-1} \mathrm{~s}^{-1}$ (at $T \approx 1.5 \mathrm{~K}$ ). The inset shows the amount of conductivity lost by the layers as a function of temperature.

the electron and hole densities are same, the single layer resistances differ by nearly a factor of 10 at $T=1.5 \mathrm{~K}$ and by a factor of 80 at $T \approx 50 \mathrm{mK}$. However the amount of conductivity $\left(\Delta \sigma_{x x}\right)$ lost by the layers as the temperature is reduced below $2 \mathrm{~K}$, does not differ by more than a factor of 2. $\Delta \sigma_{x x}$ between $2 \mathrm{~K}$ and $\approx 50 \mathrm{mK}$, is of the order of $2 \mathrm{~m} \mho$, which places it much beyond what weak localisation based effects $\left(\Delta \sigma \sim e^{2} / h \approx 40 \mu \mho\right)$ can account for 20 . In Fig. 3 we show how $d \rho / d T$ of both the electron and hole layers below $\sim 0.7 \mathrm{~K}$ turn from "metallic" to "insulating" as the carrier density of the electron layer is increased in steps, while keeping the hole density $\left(p=1.6 \times 10^{11} \mathrm{~cm}^{-2}\right)$ constant. We chose this density, such that the sheet resistivities (at $T \sim 1.5 \mathrm{~K}$ ) could be made nearly same for both the layers. The insulating state gradually evolves from a "metallic" state as the holes see more electrons. As far as we can say, the insulating state appears simultaneously in both layers. However, $n=p$ does not appear to play a particularly special role in the problem, as has been found ${ }^{15,16}$ in the context of Coulomb drag in these bilayers.

\section{DISCUSSION}

To understand what might be causing this we organise our discussion in three steps. First we have already mentioned several points which show that the physics of single layer 2DHG or 2DEG cannot explain the results. Next we consider whether any inhomogeneity caused by the large voltage bias across the $10 \mathrm{~nm}$ barrier can affect our results. Finally we discuss the possibility of the emergence of collective modes (like CDWs) in an EHBL and refer to theoretical works that suggest that 

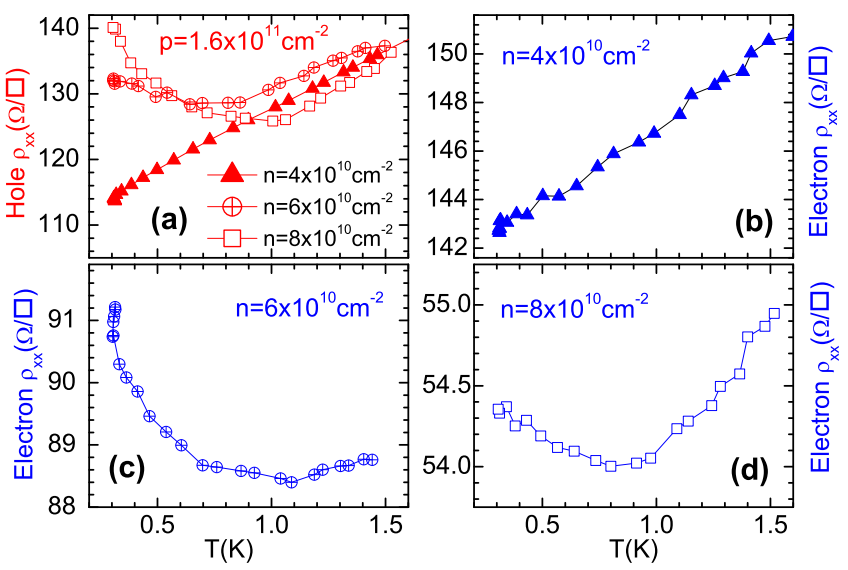

FIG. 3: Data from device E, measured in a pumped $\mathrm{He}^{3}$ cryostat. The hole density was kept fixed at $p=1.6 \times 10^{11} \mathrm{~cm}^{-2}$ while the electron density was varied in steps $\mathrm{n}=4,6,8 \times$ $10^{10} \mathrm{~cm}^{-2}$. (a) The $\rho_{x x}(T)$ for holes is "metallic" when the number of electrons is $n=4 \times 10^{10} \mathrm{~cm}^{-2}$ on the other side of the barrier. As the electron density is increased, the $\rho_{x x}(T)$ of the holes becomes insulating. (b), (c) \& (d) show the $\rho_{x x}(T)$ of the electrons. Note that the electrons are metallic at low density and insulating at the two higher densities, $k_{F} l \approx 170$ or higher for all the traces.

such modes are more likely in EHBLs than $2 \times 2$ DEGs or $2 \times 2$ DHGs.

Let us now consider the possible effect of some variation in thickness of the barrier, resulting in spatial variation of the interlayer capacitance. The question here is: In our devices does this lead to coexisting low density (insulating) regions and high density (metallic) regions? If we assume a variation of a monolayer $(\approx 0.5 \mathrm{~nm})$, this would amount to a maximum of $5 \%$ fluctuation. The corresponding density fluctuations would not (for example) be able to force regions with $p=9 \times 10^{10} \mathrm{~cm}^{-2}$ and regions with $p \approx 5 \times 10^{10} \mathrm{~cm}^{-2}$ (which is where we find the 2D MIT in the 2DHG when no 2DEG is present) in the same sample to coexist. Unless the inhomogeneity is so strong that the low density regions become strongly insulating, it cannot be the driving factor. If the $2 \mathrm{DHG}$ did become strongly inhomogeneous, it would have increased the sheet resistance at high temperatures as well. As long as the underlying idea is based on the general (single layer) picture of insulating behaviour above $h / e^{2}$ and metallic behaviour below this resistance, it is not possible to explain the results. A fundamentally different mechanism that can generate an insulating state at $\rho_{x x}<<h / e^{2}$ must be sought here.

We look for an approach that specifically takes into account that in a bilayer there can be collective modes that have no analogue in single layer situation. Collective modes are zeros of the dielectric function $\epsilon(q, \omega)$ where a system can develop density modulations without an external perturbation. For a bilayer $\epsilon(q, \omega)$ is a $2 \times 2$ matrix, whose determinant would be zero $\underline{21,22}$. Equivalently, the charge susceptibility of the system would diverge at these points. If such a mode occurs at $\omega=0$, it can be a CDW or WC phase, depending on the wavevector at which the divergence occurs. In a bilayer an electron "sees" another electron (or hole) only 10nm away, without paying a kinetic (Fermi) energy cost. As a consequence bilayer systems can give rise to interaction-driven phases more easily than single layers. In fact, existing calculations $\underline{6,8}$ of the bilayers for particle densities corresponding to $r_{s} \sim 1-10$ mention the possibility that a divergence in the eigenvalues of the bilayer $\epsilon(q, \omega=0)$ matrix is easier to get in an EHBL compared to $2 \times 2$ DEGs or $2 \times 2$ DHGs. This point needs attention because experimental data from $2 \times 2$ DEGs or $2 \times 2$ DHGs with a barrier separation of $10 \mathrm{~nm}$ and densities as low as $1 \times 10^{10} \mathrm{~cm}^{-2}$ have been available for some time ${ }^{23}, 24$. These samples had similar electron mobilities as the EHBLs reported here, but higher hole mobilities. The principal interest with these devices has been focussed on the $\nu=\frac{1}{2}+\frac{1}{2}$ bilayer Quantum Hall state, but interestingly no collective mode at $\mathrm{B}=0$ has been reported in these $\frac{25}{}$. Following Liu et $a l, \underline{\underline{6}}$ we consider the expression for eigenvalues of the susceptibility matrix, one of which must diverge to support a CDW. $\chi_{ \pm}(q)=\frac{2}{\chi_{e}^{-1}+\chi_{h}^{-1} \pm \sqrt{\left(\chi_{e}^{-1}-\chi_{h}^{-1}\right)^{2}+4\left\{\left(1-G_{e h}(q)\right) V_{e h}(q)\right\}^{2}}}$. $\chi_{e, h}$ are the single layer responses to the external potential and $V_{e h}(q)=-\frac{2 \pi e^{2}}{q} \exp (-q d)$ is the Fourier transform of the interlayer Coulomb potential. The crucial role is played by the local field correction $G_{e h}(q)$, often calculated using the Singwi-Tosi-Land-Sjölander (STLS) approximation ${ }^{26}$ that relates the local field to the structure factor. The interlayer local field correction is larger in magnitude in case of an attractive potential ${ }^{6.8}$. Due to this a divergence of the in-phase mode in an EHBL is easier to get than the corresponding divergence in the out-of-phase mode in a $2 \times 2$ DEG or a $2 \times 2$ DHG. At the same time the strong peak in $\chi(q)$, (that signals the formation of a CDW state) has been found to be strongly temperature dependent, in numerical studies 28 . The range of temperatures at which the rapid increase in resistance is seen is consistent with the expectation that it should be at a temperature smaller than the Fermi temperature of the layer with heavier mass $\left(T_{F} \approx 5 \mathrm{~K}\right.$ for holes, at $p=9 \times 10^{10} \mathrm{~cm}^{-2}$ ).

Path Integral Monte Carlo simulation of a spatially separated 2-component plasma has been carried out by

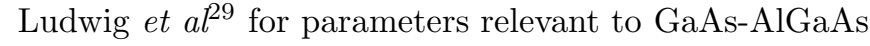
at low temperatures. The real space distribution of the holes (in presence of electrons) started developing density modulations, while the lighter electron liquid remained in a much more homogeneous state. Such a situation is quite consistent with our observations of an insulating behaviour of the holes, and a less pronounced (but clearly discernible) effect on the electrons. A density modulation (at the same wavevector) with larger amplitude in the hole layer and smaller amplitude in the electron layer is also supported by the STLS 
calculations ${ }^{8}$.

There is nothing in our observations that rules out a much awaited excitonic state in electron-hole bilayers. The interlayer pair correlation in an excitonic (bound) state would involve a more drastic modification of the structure factor and the local field corrections, compared to that of a CDW state. A CDW may well be a precursor to an excitonic state. 28

Finally, we observe that a CDW may also be a possible mechanism behind the temperature dependent enhancement in Coulomb drag reported recently ${ }^{15,16}$. We consider a situation where the densities $(n, p)$ in each layer undergo an in-phase modulation $\delta$ with a long wavelength (i.e. small $q / k_{F}$ ), such that the densities in successive half periods are $n-\delta$ and $n+\delta$. We assume that we can evaluate the contribution to drag over each half $c y$ cle and add the voltages in series. Over each half cycle the contribution is still assumed to be proportional to $1 /(n p)^{3 / 2}$, where $n, p$ are now the local densities 27 . Thus the contribution of two adjacent half cycles would be $\propto \frac{1}{(n+\delta)^{3 / 2}(p+\delta)^{3 / 2}}+\frac{1}{(n-\delta)^{3 / 2}(p-\delta)^{3 / 2}}$. Keeping terms upto $\delta^{2}$ we get the contribution from each CDW period to be $\propto \frac{2}{(n p)^{3 / 2}} \frac{1+(9 / 4) \delta^{2} / n p+(3 / 8) \delta^{2}\left(1 / n^{2}+1 / p^{2}\right)}{\left(1-\delta^{2} / n^{2}\right)^{3 / 2}\left(1-\delta^{2} / p^{2}\right)^{3 / 2}}$. This quantity is clearly larger than the unperturbed contribution $\frac{2}{(n p)^{3 / 2}}$, and it is clearly temperature dependent, since the amplitude of a CDW (i.e $\delta$ ) can increase as the temperature decreases. This argument neither depends on the exact form of the power law nor the matching of the densities. The fact that it is of order $\delta^{2}$ may have some significance in explaining why the observed effect ${ }^{15,16}$ is rather small and not a large peak or divergence expected at matched densities from an excitonic phase.

Acknowledgements: The authors acknowledge useful discussions with A.R. Hamilton and D. Neilson and J. Waldie. The work was funded by EPSRC, U.K.
* Electronic address: kd241@cam.ac.uk

$\dagger$ Present address: Department of Electronic and Electrical Engineering, University College, London, WC1E7JE

1 Y.E. Lozovik and V. I. Yudson, Sov. Phys. J.E.T.P. 44, 389 (1976)

2 G. Vignale and A.H. MacDonald, Phys. Rev. Lett., 76, 2786 (1996)

3 S. Conti, G. Vignale and A.H. MacDonald, Phys. Rev. B, 57, R6846 (1998)

4 B. Y.-K. Hu, Phys. Rev. Lett., 85, 820 (2000)

5 A.V. Balatsky, Y.N. Joglekar and P.B. Littlewood, Phys. Rev. Lett. 93, 266801 (2004)

${ }^{6}$ L. Liu et al., Phys. Rev. B, 53, 7923 (1996)

7 S. De Palo, F. Rapisarda and G. Senatore, Phys. Rev. Lett. 88, 206401 (2002)

${ }^{8}$ R.K. Moudgil, G. Senatore and L.K. Saini, Phys. Rev. B 66, 205316 (2002). R.K. Moudgil, J.Phys.: Condens. Matter 18,1285 (2006).

9 Y.N. Joglekar, A.V. Balatsky and S. Das Sarma, Phys. Rev. B 74, 233302 (2006)

10 P. Pieri, D. Neilson and G.C. Strinati, Phys. Rev. B 75, 113301 (2007)

11 J.A. Keogh et al., Appl. Phys. Lett. 87, 202104 (2005)

12 J.A. Seamons et al., Appl. Phys. Lett. 90, 052103 (2007)

13 A.F. Croxall et al., Journal of Applied Physics 104, 113715 (2008) I Farrer et al., Journal of Crystal Growth, 311, 1988 (2009)

14 U. Sivan, P.M. Solomon and H. Shtrikman, Phys. Rev. Lett. 68, 1196 (1992)

15 A.F. Croxall et al., Phys. Rev. Lett. 101, 246801 (2008)

16 J.A. Seamons et al., Phys. Rev. Lett. 102, 026804 (2009). C.P. Morath et al., Phys. Rev. B 79, 041305(R) (2009)
17 S. Das Sarma and E.H. Hwang, Phys. Rev. B 71, 195322 (2005). R. Pillarisetty et al., Phys. Rev. Lett. 90, 226801 (2003)

18 Z. Wang, Y. P. Chen, L. W. Engel, D.C. Tsui, E. Tutuc and M. Shayegan, Phys. Rev. Lett. 99, 136804 (2007)

19 C.P. Morath, J.A. Seamons, J. L. Reno and M. P. Lilly, Phys. Rev. B $\mathbf{7 8} 115318$ (2008)

20 G. Bergman, Physics Reports 107,1 p1-58 (1984)

21 S. Das Sarma and A. Madhukar, Phys. Rev. B 23, 805 (1981)

22 B. Y.-K. Hu and J. W. Wilkins, Phys. Rev. B 43, 14009 (1991)

23 M.Kellogg et al., Phys. Rev. Lett., 93,36801 (2004)

24 E. Tutuc, M. Shayegan and D.A.Huse, Phys. Rev. Lett., $\mathbf{9 3 , 3 6 8 0 2 ( 2 0 0 4 )}$

25 In refs ${ }^{23}, 24$ and subsequent papers, we didn't find a direct mention of how the resistivity of a single layer behaves in absence of the other layer. An experiment along these lines was done in $2 \times 2 \mathrm{DHG}$ with a $50 \mathrm{~nm}$ barrier by L.H. Ho et al Phys. Rev. B 77, 201402(R) (2008). 50nm is probably too large for collective modes to appear.

26 K.H. Singwi et al., Phys. Rev. 176, 589 (1968). For application to a bilayer system see L. Zheng and A.H. MacDonald, Phys. Rev. B, 49, 5522, (1994) and refs 6.8

27 A.P. Jauho and H. Smith, Phys. Rev. B 474420 (1993). E.H. Hwang and S. Das Sarma, Phys. Rev. B, 78, 075430 (2008)

28 L. Liu, L. Świerkowski and D. Nielson, Physica B, 249251, 594 (1998)

29 P. Ludwig, A. Filinov, Yu. E. Lozovik, H. Stolz and M. Bonitz, Contrib. Plasma Physics 47, No. 4-5, 335 (2007) 